\title{
Why Have Organelles Retained Genomes?
}

\author{
John F. Allen ${ }^{1 *}$, William F. Martin ${ }^{2}$
}

1. Research Department of Genetics, Evolution and Environment, Darwin Building, University College London, Gower Street, London WC1E 6BT, UK

2. Institute of Molecular Evolution, Heinrich-Heine-University, 40225 Düsseldorf, Germany

* Correspondence: j.f.allen@ucl.ac.uk

Genes in mitochondria and chloroplasts are co-located with their gene products to permit regulation of trans-membrane electron transport at the energetic boundary of the cell.

"I shall attempt to consider the mechanism whereby the contact between the organism and its environment is regulated, particularly in relation to the functions of the membranes that form the boundary between the organism and its environment ....

The two may be regarded as equivalent phases between which dynamic contact is maintained by the membranes that separate and link them." (Mitchell 1957)

In this issue of Cell Systems, Johnston and Williams (Johnston and Williams 2016) tell of incisive progress on one of the classical questions in eukaryotic cell biology, namely "why have bioenergetic organelles retained genomes?" This is an exciting topic, one that gets right to the fabric of life, because without bioenergetic organelles — chloroplasts and mitochondria — complex life would not exist. What's the story? It is this. 
Although they appear neatly tucked away in the cell, securely wrapped in membranes, the inner machineries of mitochondria and chloroplasts are the organism's front line of dynamic contact with its environment. The innermost membranes of these organelles are the cell's primary interface with the outside world, harnessing energy by means of serious chemistry - electron transfer. Light and dissolved oxygen reach the thylakoids of chloroplasts and the mitochondrial inner membrane effortlessly through the cytoplasm, glancing at surface photoreceptors and oxygen sensors. But when they reach the bioenergetic membranes deep inside organelles, electrons are set usefully in motion. These moving electrons have an essential function in generating ATP, while at the same time being highly dangerous, a live wire that can damage or kill the cell through the generation of reactive oxygen species (Lambert and Brand 2009).

Life and death of the cell depend on what's happening, and happening now, inside mitochondria and chloroplasts, at the organism's primary energetic interface with the unpredictable and unyielding outside world. This energetic interface is redox chemistry - electron transfer from a donor to an acceptor. In the light-driven photosynthetic reaction centers of plant chloroplasts, electrons are extracted first from water, making oxygen. In animal and plant mitochondria, electrons from food are transferred finally to oxygen, making water, at cytochrome oxidase of the respiratory chain. These electron transfer chains are the vital redox machines that keep us alive, second to second through each day. The innermost membranes of mitochondria and chloroplasts are where the electrons change hands and keep our lives running. This is the front line. The crucial information about the functional state of bioenergetic membranes is the redox state of their electron carriers. In mitochondria, the electron carriers of the respiratory electron transport chain are complex I, complex II, complex III and complex IV (Figure 1), all connected by the mobile carriers ubiquinone and cytochrome $c$. It is not enough for the cell to know how much food or oxygen may be on its way. Direct sensing of the redox state of the carriers in the membrane is required in real time. For this, only redox sensors will do.

Almost all proteins of chloroplasts and mitochondria are encoded in the cell nucleus and imported, as precursors, by the protein import machineries germane to each organelle (Paul et al. 2013). Yet chloroplasts and mitochondria possess their own DNA. Why? Both chloroplasts and mitochondria themselves encode protein 
components of the electron carriers of their electron transport chains. Moreover, chloroplasts and mitochondria encode almost exclusively proteins of their electron transport chains, together with proteins and RNAs of the ribosomes needed to synthesize them (Maier et al. 2013). The key redox proteins at the front line of cell metabolism (Figure 1) contain subunits with different sites of synthesis, and their blueprints are housed in different genomes. It is a curious and untidy arrangement. Why this redundancy? And why does any gene at all remain in organelles, given that around $99 \%$ of organellar proteins are now encoded by evolutionary exports to the nucleus (Allen 2015)? If any gene could move, why didn't they all? In short, why do mitochondria and chloroplasts contain DNA?

Johnson and Williams address the question by analyzing every logically possible combination of the 65 protein-coding genes known and annotated for mitochondrial DNA from 2,015 species. Of this huge set of possibilities, only 74 distinct combinations exist today. What is their evolutionary relationship? How can selective gene loss account for it, and in which order were genes and gene combinations lost, incrementally, from the ancestral endosymbiont?

Using a novel algorithm - HyperTraPS - for sampling rare evolutionary paths on a hypercubic transition network, Johnson and Williams calculate probabilities for each gene's loss, and for its replacement with each and every other gene. The results show that the genes least likely to be lost are those that encode core subunits of proteins of the respiratory electron transport chain. From known structures, usually from X-ray crystallography, these proteins have the strongest predicted interaction with neighboring subunits, suggesting that regulation of their quantities determines the rate of assembly of whole respiratory chain complexes. In short, genes are in mitochondrial DNA in order that they can be regulated.

This conclusion is consistent with the "CoRR" Hypothesis - organelle genes are $\underline{C o}-$ located with their gene products to allow $\underline{R}$ edox $\underline{R}$ egulation of their expression (Allen 2015). Imagine a cell with a hundred mitochondria having no DNA of their own, and with all of the genes for organelle electron transport chain components being in the nucleus. Without organelle DNA, the cell would have a life-threatening regulatory 
problem. How so? Suppose one mitochondrion needs more complex III to keep its electrons flowing smoothly and to minimize production of reactive oxygen species. It could signal to the nucleus that it needed more complex III, and the nucleus would respond, providing more complex III precursors to the cytosol. The single, wanting mitochondrion is rescued, but the other ninety-nine now complain that there is too much complex III and something else needs adjustment, electron flow in mitochondria would go haywire, and the cell is toast. By contrast, if a component of each key complex in the electron transport chain is encoded in mitochondrial DNA, each mitochondrion can react individually to the needs of its own electron transport chain by expressing what is needed where it is needed. This does not require the organelle to encode each entire complex, just a component of each that sets the pace for the complex's assembly. And this is exactly what Johnston and Williams (Johnston and Williams 2016) show.

For chloroplasts, the CoRR hypothesis has substantial direct experimental support. Reaction centers in photosynthesis receive absorbed excitation energy from lightharvesting pigment-proteins, and convert this energy to transmembrane electron transfer through a series of electron carriers intrinsic to the chloroplast's inner membrane. All these components are proteins. Redox control of transcription acts on reaction center genes in chloroplast DNA, and works by a typically bacterial twocomponent system whose mechanistic details are becoming clear (Puthiyaveetil et al. 2013). A cyanobacterial homologue of the chloroplast sensor kinase shows regulated phosphoryl group transfer to two transcriptional response regulators (Ibrahim et al. 2016). Application of HyperTraPS to chloroplast gene content would be a reference point for this technique, and might perhaps harbor some surprises for plant evolution.

For mitochondria, there is no evidence against CoRR, but less is known about mechanisms of their redox regulation of gene expression. The common feature of proteins made in mitochondria is often thought to be their hydrophobicity. But hydrogenosomes, anaerobic forms of mitochondria, are chock-full of hydrophobic membrane proteins and lack mtDNA completely (Müller et al. 2012). How so? Hydrogenosomes generate ATP by fermentations: no electron transport chain, no organelle DNA, despite abundant hydrophobic proteins. For animal mitochondria, 
gene expression is now known to be redox regulated, but by an unexpected mechanism involving the mitochondrial topoisomerase (Sobek et al. 2013).

Mitochondria and chloroplasts remain true to their prokaryotic ancestry - they are energetically diverse, versatile, adaptable, and instantly responsive to environmental change. It seems that these life-supporting smart-organelles retain genomes and genetic systems for exactly this reason. Johnston and Williams make a connection between the genes in mitochondrial DNA and the components that can become ratelimiting for assembly of respiratory chain complexes. This makes a lot of sense when seen from the standpoint of mitochondrial redox chemistry. Gene-level regulation of the electron current keeps our cells supplied with ATP, and keeps us alive.

\section{Acknowledgements}

J.F.A. thanks The Leverhulme Trust for Emeritus Research Fellowship EM-2015-068. W.F.M. thanks the European Research Council for funding.

\section{References}

Allen JF (2015) Why chloroplasts and mitochondria retain their own genomes and genetic systems: Colocation for redox regulation of gene expression P Natl Acad Sci USA 112:10231-10238 doi:10.1073/pnas.1500012112

Ibrahim IM, Puthiyaveetil S, Allen JF (2016) A two-component regulatory system in transcriptional control of photosystem stoichiometry: redox-dependent and sodium ion-dependent phosphoryl transfer from cyanobacterial histidine kinase Hik2 to response regulators Rre1 and RppA Frontiers in Plant Science 7 doi:10.3389/fpls.2016.00137

Johnston IG, Williams BP (2016) Evolutionary inference across eukaryotes identifies specific pressures favoring mitochondrial gene retention Cell Systems 2:x-y

Lambert AJ, Brand MD (2009) Reactive Oxygen Species Production by Mitochondria. In: Stuart JA (ed) Mitochondrial DNA: Methods and Protocols. Humana Press, Totowa, NJ, pp 165-181. doi:10.1007/978-1-59745-521-3_11 
Maier UG, Zauner S, Woehle C, Bolte K, Hempel F, Allen JF, Martin WF (2013)

Massively convergent evolution for ribosomal protein gene content in plastid and mitochondrial genomes Genome Biol Evol 5:2318-2329 doi:Doi $10.1093 / \mathrm{Gbe} / \mathrm{Evt} 181$

Mitchell P The Origin of Life and the Formation and Organising Functions of Natural Membranes. In: Oparin A (ed) International Symposium on the Origin of Life, Moscow, 1957. Publishing House of the Academy of Sciences, USSR, pp 229234

Müller M et al. (2012) Biochemistry and evolution of anaerobic energy metabolism in eukaryotes Microbiology and Molecular Biology Reviews 76:444-495 doi:10.1128/mmbr.05024-11

Paul P, Simm S, Blaumeiser A, Scharf K-D, Fragkostefanakis S, Mirus O, Schleiff E (2013) The protein translocation systems in plants - composition and variability on the example of Solanum lycopersicum BMC Genomics 14:1-16 doi:10.1186/1471-2164-14-189

Puthiyaveetil S, Ibrahim IM, Allen JF (2013) Evolutionary rewiring: a modified prokaryotic gene-regulatory pathway in chloroplasts Philosophical Transactions of the Royal Society B-Biological Sciences 368 doi:10.1098/rstb.2012.0260

Sobek S et al. (2013) Negative regulation of mitochondrial transcription by mitochondrial topoisomerase I Nucleic Acids Research 41:9848-9857 doi:10.1093/nar/gkt768

Figure Legend

Figure 1. Genes in the mitochondrion are co-located with their gene products.

Membrane-intrinsic cytochrome oxidase, respiratory complex IV, dumps onto oxygen $\left(\mathrm{O}_{2}\right)$ the electrons $\left(\mathrm{e}^{-}\right)$it receives from a chain of respiratory electron carriers, almost all of which are proteins intrinsic to the mitochondrial inner membrane. All respiratory electron transport chain complexes contain some protein subunits, shown in gold, that are encoded and transcribed in the cell nucleus, translated on ribosomes 
in the cytosol, and then imported into the mitochondrion as precursors. Respiratory chain complexes I, II, III and IV and the coupling ATPase also contain a core of protein subunits, shown in red-brown, that are encoded and completely synthesized entirely within the mitochondrion, starting with transcription of mitochondrial DNA (mtDNA). Redox regulation is feedback from the redox state of respiratory electron carriers and governs mitochondrial transcription. The composition of the electron transport chain thereby regulates itself in response to variation in the supply of electron donors and acceptors. The terminal respiratory electron acceptor is molecular oxygen, $\mathrm{O}_{2}$. The rate of synthesis of each whole respiratory complex is determined by the rate of synthesis of its mitochondrially-encoded subunits. The stoichiometry of subunits can be changed to match prevailing redox conditions determined by metabolism, by available electron sources and sinks, and by rate of ATP synthesis. 